Revue d'histoire de l'Amérique française

REVUE D.HISTOIRE DE L'AMÉRIQUE FRANÇAISE

\title{
La scolarisation élémentaire en Mauricie (1850-1900) : esquisse de la population scolaire et des résultats de ses apprentissages
}

\author{
Jocelyne Murray
}

Volume 55, numéro 4, printemps 2002

URI : https://id.erudit.org/iderudit/010442ar

DOI : https://doi.org/10.7202/010442ar

Aller au sommaire du numéro

Éditeur(s)

Institut d'histoire de l'Amérique française

ISSN

0035-2357 (imprimé)

1492-1383 (numérique)

Découvrir la revue

Citer cet article

Murray, J. (2002). La scolarisation élémentaire en Mauricie (1850-1900) :

esquisse de la population scolaire et des résultats de ses apprentissages. Revue d'histoire de l'Amérique française, 55(4), 573-601.

https://doi.org/10.7202/010442ar
Résumé de l'article

Cet article traite de la scolarisation élémentaire dans les circonscriptions de Champlain, de Maskinongé et de Saint-Maurice pendant la deuxième moitié du XIXe siècle. L'étude montre qu'il faut attendre la fin du siècle pour que la presque totalité des enfants âgés entre sept et treize ans fréquentent l'école. Cependant, les écoliers affichent une faible assiduité tout au long de la période, sauf dans les écoles modèles et académiques. De multiples facteurs peuvent expliquer l'irrégularité de la présence en classe, surtout dans les " petites écoles » disséminées sur tout le territoire. À la pauvreté et aux corvées familiales incontournables s'ajoutent la non-obligation scolaire et le manque de formation en pédagogie du personnel enseignant. Et toutefois,

l'apprentissage des matières de base, de morcelé qu'il était au milieu du siècle, devient plus complet les décennies suivantes. Si bien qu'à la fin du siècle, les élèves apprennent simultanément la lecture, l'écriture et le calcul. Pour expliquer ces évolutions discordantes, force est de revenir sur les explications ci-dessus et de proposer de nouvelles pistes de recherche.
Tous droits réservés @ Institut d'histoire de l'Amérique française, 2000
Ce document est protégé par la loi sur le droit d'auteur. L'utilisation des services d'Érudit (y compris la reproduction) est assujettie à sa politique d'utilisation que vous pouvez consulter en ligne.

https://apropos.erudit.org/fr/usagers/politique-dutilisation/ 


\section{La scolarisation élémentaire}

en Mauricie (I850-1900) : esquisse de la population scolaire et des résultats de ses apprentissages ${ }^{\mathrm{I}}$

\section{JOCELYNE MURRAY}

Centre interuniversitaire d'études québécoises Université du Québec à Trois-Rivières

RÉSUMÉ - Cet article traite de la scolarisation élémentaire dans les circonscriptions de Champlain, de Maskinongé et de Saint-Maurice pendant la deuxième moitié du XIXe siècle. L'étude montre qu'il faut attendre la fin du siècle pour que la presque totalité des enfants âgés entre sept et treize ans fréquentent l'école. Cependant, les écoliers affichent une faible assiduité tout au long de la période, sauf dans les écoles modèles et académiques. De multiples facteurs peuvent expliquer l'irrégularité de la présence en classe, surtout dans les «petites écoles » disséminées sur tout le territoire. À la pauvreté et aux corvées familiales incontournables s'ajoutent la non-obligation scolaire et le manque de formation en pédagogie du personnel enseignant. Et toutefois, l'apprentissage des matières de base, de morcelé qu'il était au milieu du siècle, devient plus complet les décennies suivantes. Si bien qu'à la fin du siècle, les élèves apprennent simultanément la lecture, l'écriture et le calcul. Pour expliquer ces évolutions discordantes, force est de revenir sur les explications ci-dessus et de proposer de nouvelles pistes de recherche.

ABSTRACT - This article deals with the pupils enrolled at the elementary schools level in the counties of Champlain, Maskinongé, and Saint-Maurice during the second part of the Nineteenth Century. The study of the school population shows that by the end of the century almost all children between seven and thirteen years of age attended school. However, with the exception of model and academic schools, such progress was undermined by the irregular presence in class, particularly in the "small schools" scattered around the countryside. Many elements can explain the situation : to the poverty or to

1. Je remercie René Hardy, pour avoir relu ce texte et pour ses judicieux commentaires, ainsi que les évaluateurs anonymes de la revue. Ma gratitude va également au Fonds FCAR, à la Fédération canadienne des femmes diplômées (Bourse Georgette Lemoyne) et à l'Université du Québec à Trois-Rivières pour leur soutien financier. 
the inevitable family chores, can be added the lack of compulsion and the poor quality of teaching. And yet, the learning of the basic subjects, which was discontinuous by the mid$19^{\text {th }}$ Century, became more complete the following decades. At the end of the century, all students would finally learn simultaneously how to read, write, and calculate. Such an evolution questions the accuracy of the above explanations and implies new hypotheses.

\section{INTRODUCTION}

Dans le vaste champ de l'histoire de l'éducation, le terrain de l'histoire locale traitant de l'enseignement élémentaire commence à être défriché, les historiens ayant surtout étudié, dans ses résultats globaux, l'engagement de l'État et la participation du clergé en vue d'établir et de faire fonctionner le système d'instruction publique. La question de la scolarisation au niveau local a été abordée par des chercheurs comme Andrée Dufour, qui analyse la genèse des différentes lois scolaires en regard des vœux exprimés par les collectivités, J. I. Little, dans ses travaux sur l'organisation de la société dans les Cantons de l'Est, Gérard Bouchard, qui s'est penché sur l'évolution de l'alphabétisation au Saguenay, et Jacques Ouellet, qui a étudié la scolarisation dans la même région entre 1876 et $1966^{2}$. Notons enfin que maints travaux d'histoire régionale renferment des études le plus souvent descriptives des progrès accomplis par l'enseignement.

Dans l'ensemble, l'éducation en milieu régional a été abordée dans ses aspects institutionnels et statistiques. Nous avons l'intention de suivre cette voie, question d'apporter des éléments supplémentaires de comparaison

2. Andrée Dufour, Tous à l'école. État, communautés rurales et scolarisation au Québec de 1826 à 1859 (Montréal, HMH, 1996), ainsi que La scolarisation au Bas-Canada, 1826-1859 : une interaction Étatcommunautés locales, thèse de doctorat (histoire), Université du Québec à Montréal, 1992, x-398 p. J. I. Little, State and Society in Transition. The Politics of Institutional Reform in the Eastern Townships, 1838-1852 (Montréal, McGill-Queen's University Press, 1997), chap. 6 et 7, ainsi que Crofters and Habitants. Settler Society, Economy, and Culture in a Quebec Township, 1848-1881 (Montréal, McGillQueen's University Press, 1991), chap. 8. Gérard Bouchard, «Évolution de l’alphabétisation masculine au Saguenay : les variables géographiques (1842-1971)", Historical Papers/Communications historiques de la Société historique du Canada (1989) : 13-35; Gérard Bouchard et Jeannette Larouche, «Nouvelle mesure de l'alphabétisation à l'aide de la reconstitution automatique des familles», Histoire sociale/Social History, 22,43 (mai 1989) : 91-119; Gérard Bouchard et Raymond Roy, "Fécondité et alphabétisation au Saguenay et au Québec (XIX et XX ${ }^{\mathrm{e}}$ siècles)", Annales de démographie historique (1991) : 173-201. Dans la même optique, il y a également l'article de Marc StHilaire, «Mobilité et alphabétisation au Saguenay, 1840-1940», dans Serge Courville et Normand Séguin, dir., Espace et culture (Sainte-Foy, Les Presses de l’Université Laval, 1995), 227-236. Jacques Ouellet, L'Instruction publique au Saguenay (1876-1966) à travers les rapports du surintendant de l'instruction publique : une étude exploratoire, mémoire de maîtrise (études régionales), Université du Québec à Chicoutimi, 1984, 284 p., et «Le développement du système scolaire au Saguenay-LacSaint-Jean depuis 150 ans», dans Saguenayensia, 30,1 (janv.-mars 1988) : 6-36. 
dans l'analyse des liens entre l'école et la société. Mais également, l'approche quantitative constitue, à notre avis, la première et indispensable étape à une étude de plus grande envergure destinée à mieux évaluer la part de la socio-économie et celle du personnel enseignant dans la pénétration de l'éducation dans une région donnée.

La présente esquisse de la population scolaire de la Mauricie est extraite d'une recherche doctorale ${ }^{3}$ sur la scolarisation dans les trois circonscriptions composant cette région: Maskinongé, Champlain et Saint-Maurice. La région a pour double caractéristique d'avoir une population ancienne dans d'importants villages le long du fleuve, comme Yamachiche ou Batiscan, vivant d'agriculture et de petites entreprises rurales, et un peuplement plus récent (1850-1890) dans les paroisses et les villages plus nombreux de l'arrière-pays et axés sur l'exploitation forestière et la sidérurgie. Puis, à l'embouchure du Saint-Maurice, se profile la ville de Trois-Rivières, centre administratif et commercial. Entre les trois circonscriptions existent quelques différences. Et d'abord, celle de Maskinongé est plus axée que les autres sur l'agriculture, encore que la forêt n'y joue pas un rôle négligeable. Celles de Saint-Maurice et de Champlain connaissent un développement plus tardif de leur arrière-pays que la précédente, un développement qui mènera toutefois à l'urbanisation et à l'industrialisation de la vallée mauricienne à partir de la seconde moitié des années 1880 .

À ces différences régionales s'en ajoutent d'autres de type socioprofessionnel. La Mauricie est peuplée d'agriculteurs prospères exploitant des terres ancestrales établies le long du fleuve Saint-Laurent et de défricheurs de nouveaux territoires; il y a aussi des artisans et des professionnels installés au cœur des villages ainsi que des ouvriers des industries rurales et, finalement, des citadins. Il faudra tenir compte de ces différences dans l'étude des attentes des contribuables face à la scolarisation.

C'est à la lumière de ces particularités que nous aimerions examiner la scolarisation ${ }^{4}$. Les corporations scolaires des circonscriptions de Champlain, de Maskinongé et de Saint-Maurice ont en effet élaboré un réseau assez important d'écoles. Et cependant, comme ailleurs au Québec, la scolarisation de la totalité des enfants ne s'est pas accomplie instantanément ni complètement.

3. Jocelyne Murray, La scolarisation au Québec (1850-1900), l'exemple de la Mauricie, thèse de doctorat (études québécoises), Université du Québec à Trois-Rivières, 1999, xvi-498 p.

4. Cet article ne concerne que les écoles catholiques de la Mauricie, les écoles et les effectifs protestants étant trop peu nombreux. En effet, il n'y a que 2 corporations protestantes dans la région, à Sainte-Ursule et à Trois-Rivières. 


\section{I - L'ORGANISATION SCOLAIRE DU QUÉBEC}

Pour bien comprendre les pages qui suivent, il importe de rappeler dans ses grandes lignes l'organisation scolaire du Québec tout au long du xix ${ }^{\mathrm{e}}$ siècle. Selon les différents programmes d'études en vigueur, les écoles dispensant le cours primaire sont réparties en trois niveaux : les écoles élémentaires, vouées strictement à l'enseignement dit élémentaire ${ }^{5}$, les écoles modèles, qui accueillent également des écoliers du cours élémentaire, ainsi que les académies, qui regroupent des élèves des trois niveaux. Il n'existe pas à proprement parler de cours secondaire dans le réseau public. À la place, il se donne un "cours supérieur», qui prolonge le cours élémentaire, et qui est assumé par les écoles modèles et académiques. Les élèves vont y parfaire leur formation de base en vue de la pratique d'un métier dans le commerce et dans l'enseignement. Le programme du cours académique se distingue par un enseignement plus poussé en sciences (mathématiques, physique et chimie) et en histoire; on y aborde la littérature et parfois l'étude du latin 6 .

La loi scolaire de 1846 prescrit aux commissaires d'école de voir à l'organisation pédagogique des trois catégories d'écoles. En général, ces derniers en confient la responsabilité au personnel enseignant. Ainsi, chaque corporation, voire chaque enseignant, détermine son propre programme d'étude. Le Guide de l'instituteur de F.-X. Valade (1851) et le Cours de pédagogie de Jean Langevin (1865) servent de référence aux instituteurs, de même que la liste des questions pour l'examen des candidats aux brevets publiée dans le Journal de l'Instruction publique (1862) ${ }^{7}$. À partir de 1860, le conseil de l'Instruction publique dresse une liste de livres approuvés, imposant une certaine limite dans le choix des manuels.

5. Du moins en théorie. Il arrive, en effet, que des élèves fassent leurs études de niveau modèle dans une école élémentaire. Mais de tels cas demeurent exceptionnels.

6. Michel Allard et Bernard Lefebvre, dir., Les programmes d'études catholiques francophones $d u$ Québec. Des origines à aujourd'hui (Montréal, Les Éditions Logiques, 1998), 130.

7. François-Xavier Valade, Guide de l'instituteur contenant une série de réponses aux questions insérées dans la circulaire numéro douze du surintendant de l'éducation sur les diverses branches d'instruction prescrites par la loi des écoles en opération dans le Bas-Canada. Ces questions et ces réponses forment maintenant un seul et même ouvrage destiné à l'usage des écoles (Montréal, J. B. Rolland, Imprimeurlibraire, 1853), 288 p., $3^{\mathrm{e}}$ édition; Jean Langevin, Abbé, Cours de pédagogie ou principes d'éducation (Québec, C. Darveau, Imprimeur, 1865), 409 p.

8. Thérèse Hamel, «Les programmes d'études de 1861 à 1923 », dans Michel Allard et Bernard Lefebvre, dir., Les programmes d'études catholiques francophones du Québec. Des origines à aujourd'hui (Montréal, Les Éditions logiques, 1998), 45-311; Jean-Pierre Charland, L'entreprise éducative au Québec, 1840-1900 (Sainte-Foy, Les Presses de l’Université Laval, 2000), 223-228. 
Il faut attendre l'année 1872-1873 pour que le conseil de l'Instruction publique établisse enfin un premier programme officiel d'études primaires de quatre années : l'école élémentaire est répartie en deux degrés d'une année chacun, tandis que l'école modèle et l'école académique méritent une année chacune. Au total, quatre années que Louis-Philippe Audet qualifie de "théoriques", dans la mesure où le programme exige un temps autrement plus long' ${ }^{9}$. Et de fait, le conseil de l'Instruction publique modifiera souvent son programme par la suite. C'est ainsi que les changements de 1888 et de 1898 font porter la durée du cours élémentaire sur quatre ans et celle des cours modèle et académique sur deux ans chacun. Et encore, de tels remaniements sous-estiment toujours le temps qu'il faut passer sur les bancs d'école ${ }^{10}$.

\section{II - ÉCOLES ET ENSEIGNANTS EN MAURICIE}

La Mauricie a vu ses écoles tripler de 88 en 1852-1853 à 264 en 1900-1901. Parmi elles, celles strictement élémentaires formaient l'immense majorité : 81 au milieu du $\mathrm{XIX}^{\mathrm{e}}$ siècle et 234 cinquante années plus tard. Pour leur part, les écoles modèles sont passées de 6 en 1852-1853 à 21 en 1900-1901, et les écoles académiques de 1 à $9^{11}$. Ajoutons qu'en 1900-1901, 203 écoles élémentaires se trouvent en pleine campagne, contre 24 en milieu villageois et 7 à Trois-Rivières, tandis que 26 écoles modèles et académiques se situent dans 17 villages et desservent les 37 paroisses de la Mauricie, alors que 4 sont à Trois-Rivières.

D’une manière générale, il existe un important écart dans la qualité des écoles suivant qu'elles se trouvent à la campagne ou dans les villages et à Trois-Rivières. Même les écoles élémentaires de village se démarquent des écoles de rang. Elle sont habituellement les meilleures de la corporation car on y engage, sauf quelques rares exceptions, des institutrices dont la compétence est reconnue. Ces dernières reçoivent d'ailleurs une rémunération supérieure aux enseignantes œuvrant à la campagne.

9. Louis-Philippe Audet, L'histoire de l'enseignement au Québec, 1840-1971 (Montréal, Holt, Rinehart et Winston, 1971), $2: 106$.

10. Ibid., 273; T. Hamel, op.cit., 49-66.

11. Rapport annuel du surintendant de l'Instruction publique de la province de Québec pour l'année 1852-53 et ibid. pour l'année 1900-1901 (dorénavant nous utiliserons le sigle RSIP suivi de l'année). Les données proviennent, pour l'année 1852-1853, du Résumé statistique des divers rapports des inspecteurs d'écoles et du rapport de Petrus Hubert du 5 mars 1853; et pour 1900-1901, des tableaux intitulés Écoles élémentaires catholiques, 186-189, ainsi que du Résumé des rapports des collèges classiques, des académies et des écoles modèles catholiques subventionnées, 258-291. 
Par ailleurs, la grande majorité des établissements scolaires publics en Mauricie sont accessibles autant aux garçons qu'aux filles. À la campagne et dans les villages, les écoles élémentaires publiques sont, pour la plupart, des établissements mixtes : 220 le sont, seulement trois sont des écoles séparées de garçons et quatre des écoles séparées de filles. La ville de Trois-Rivières se distingue des localités rurales puisqu'on y dénombre trois écoles publiques de garçons et autant pour les filles, la seule école mixte étant située sur l'île de la Potherie ${ }^{12}$. La séparation des sexes au niveau élémentaire est une caractéristique urbaine que l'on retrouve notamment à Montréal ${ }^{13}$. De leur côté, toutes les écoles modèles et académiques sont séparées selon le sexe, à l'exclusion de 5 établissements.

Le phénomène des écoles séparées selon le sexe est une initiative du clergé. Notons cependant qu'il n'est pas unique au Québec; il serait même d'inspiration européenne ${ }^{14}$. Ainsi en France, dans les années 1880, la loi prescrit la division des écoles selon le sexe pour les communes dépassant cinq cents habitants ${ }^{15}$. C'est également une pratique courante dans les décennies 1850 et 1860 dans les écoles urbaines ontariennes (protestantes et catholiques) lorsque l'espace et le nombre d'enseignants le permettent $^{16}$. Ce qui n'est évidemment pas le cas dans la campagne mauricienne.

Pour compléter ce tableau, il convient de glisser un mot sur les enseignants. Tout d'abord, ils ont augmenté leurs effectifs à un rythme semblable à celui des écoles. En 1852-1853, on comptait en effet 83 enseignants laïques et environ 25 appartenant à un ordre religieux. Un demi-siècle plus tard, il y avait 292 laïcs et 136 frères et sœurs. Si l'on reporte ces données sur le nombre d'écoles en Mauricie, on voit que le ratio a légèrement progressé de 1,3 à 1,6 enseignant par école. Notons toutefois un important changement : la féminisation de plus en plus marquée de la profession. En 1852-1853, l'on comptait 31 hommes et 52 femmes dans le personnel laïque;

12. Archives municipales de Trois-Rivières, Rapports semestriels de la Commission scolaire de Trois-Rivières pour l'année 1898. Ce document corrige le Rapport annuel du Surintendant de l'Instruction publique de la province de Québec pour l'année 1900-1901 qui dénombre 5 écoles mixtes à Trois-Rivières.

13. Robert Gagnon, Histoire de la Commission des écoles catholiques de Montréal (Montréal, Boréal, 1996), 29-30 et RSIP.

14. David Tyack et Elisabeth Hansot, Learning Together. A History of Coeducation in American Public School (New York, Russell Sage Foundation, 1992), 4.

15. Jean Peneff, Écoles publiques, écoles privées dans l'Ouest 1880-1950 (Paris, l'Harmattan, 1987), 38-39.

16. Susan E. Houston et Alison Prentice, Schooling and Scholars in Nineteenth-Century Ontario (Toronto, University of Toronto Press, 1988), 250. 
au début du $\mathrm{xx}^{\mathrm{e}}$ siècle, le nombre d'hommes tombait à 10 , tandis que celui des femmes atteignait 282, pendant qu'œuvraient 38 frères et 98 sœurs ${ }^{17}$. Par ailleurs, la proportion des religieux a augmenté d'environ $23 \%$ dans les années 1850 à près de $32 \%$ en 1900-1901. Certes, ils ne dominent pas la scène, comme on l'a trop souvent prétendu, mais leur présence est de plus en plus palpable. Et si l'on répartit le personnel entre les catégories d'écoles, surgit un phénomène intéressant : au début du $\mathrm{xx}^{\mathrm{e}}$ siècle, les écoles élémentaires sont animées par 265 enseignants laïques et seulement 10 religieuses, tandis que les écoles modèles et académiques sont sous la responsabilité de 27 enseignants laïques et de 126 religieux. Donc, si l'école élémentaire est du ressort des laïcs, les écoles modèles et académiques sont dominées par les frères et les sœurs. Ajoutons à cette distinction une autre différence essentielle : en 1900-1901, on compte en moyenne 1,2 enseignant par école élémentaire, contre 5,1 par école modèle ou académique.

Au total, le système scolaire mauricien favorise nettement le monde villageois et trifluvien sur celui des campagnes, bien que ce dernier accueille la grande majorité des écoles.

\section{III - ÉTAT DE LA POPULATION SCOLAIRE}

Dans les rapports annuels du surintendant, on évalue de deux manières la population scolaire : par le nombre d'élèves inscrits chaque année et par la présence moyenne des écoliers en classe durant cette même période. C'est sous le terme "fréquentation» que nous désignons le nombre des élèves inscrits. Les effectifs peuvent paraître surévalués, voire trompeurs, car ils ne rendent pas compte de la présence régulière en classe. Ils reflètent néanmoins une prise de contact minimal avec l'école, quel que soit le temps consacré à la scolarisation. Par ailleurs, nous utilisons le terme "assiduité» pour faire référence à la présence régulière des élèves en classe. Cette mesure, aussi appelée "assistance moyenne des élèves ${ }^{18}$ ", permet d'évaluer non seulement l'impact de l'école mais l'intérêt qu'elle éveille, les absences pouvant, a contrario, être perçues comme un désaveu ou de

17. RSIP 1852-1853. Voir appendice du onzième volume des journaux de l'Assemblée législative de la Province du Canada Session 1852-53 : Rapport de Petrus Hubert, inspecteur d'écoles, Yamachiche, 5 mars 1853, 51. RSIP 1900-1901, Instituteurs et institutrices laïques pour les écoles élémentaires catholiques, 194-197 et pour les écoles modèles et académiques catholiques, 214-215. Le dénombrement des enseignants religieux provient du Résumé des rapports des collèges classiques, des académies et des modèles catholiques subventionnées, 258 et ss. Récapitulation des statistiques scolaires de la Province, 246-249.

18. Terme utilisé dans les tableaux statistiques des rapports annuels du surintendant. 
l'indifférence de la part de la population face à l'instruction. L'information sur l'assiduité apparaît régulièrement à compter de 1873-1874, sauf pour une brève interruption de 1891 à 1893 . Nous examinerons successivement la fréquentation et l'assiduité.

\section{A - La fréquentation}

\section{I - Données globales}

Durant l'année 1850-1851, 3476 élèves fréquentent les écoles de la Mauricie ${ }^{19}$. Il est fort possible que cette évaluation sous-estime la réalité. Le surintendant Jean-Baptiste Meilleur explique, en effet, que les données fournies par les corporations scolaires lui semblent incomplètes ${ }^{20}$. Cette difficulté s'atténue avec l'instauration de l'inspectorat, puisque les fonctionnaires recenseront eux-mêmes annuellement les élèves. De plus, ils apporteront des précisions additionnelles dans le rapport accompagnant ces statistiques. Le tableau 1 illustre, à compter de 1852-1853, l'évolution décennale de la population scolaire.

Entre 1852 et 1872, le nombre des élèves a plus que doublé, tandis que leur proportion par rapport à la population totale gagne $6 \%$. On peut constater que, pendant les vingt années suivantes, la croissance est plus modeste et le pourcentage des élèves par rapport à la population totale n'augmente que de $1 \%$. Dans les années 1890 , on assiste à la plus forte croissance du nombre d'écoliers jamais enregistrée, soit près de 3500 . En cinquante ans, le nombre a été multiplié par 3,3 tandis que la population ne double pas tout à fait.

Le ralentissement de l'expansion de la population scolaire entre les années 1870 et 1890 est une conséquence directe des difficultés économiques doublées d’un exode sans précédent qui provoque le départ de milliers d'habitants ${ }^{21}$. Les rapports des inspecteurs d'écoles des trois circonscriptions de la Mauricie soulignent, dès 1873-1874 et à maintes reprises par

19. RSIP 1850-1851. La Mauricie est alors composée de deux circonscriptions : Champlain et SaintMaurice. La population scolaire est évaluée pour la deuxième partie de l'année 1850, à 3494 élèves et, pour la première partie de 1851, à 3458 élèves. Nous avons fait une moyenne de ces deux totaux.

20. RSIP pour 1850 publié le 8 septembre 1852 .

21. René Hardy et Normand Séguin, Forêt et société en Mauricie (Montréal, Boréal Express, 1984), 138; Yolande Lavoie, L'émigration des Canadiens aux États-Unis avant 1930. Mesure du phénomène (Montréal, Presses de l'Université de Montréal, 1972), 58-64; et L'émigration des Québécois aux États-Unis de 1840 à 1930 (Québec, Éditeur officiel, 1979), 43. La circonscription de Berthier, limitrophe de celle de Maskinongé, a aussi été affectée par ce flot d'émigration. Voir Bruno Ramirez, Par monts et par vaux. Migrants canadiens-français et italiens dans l'économie nord-atlantique, 1860-1914 (Montréal, Boréal, 1991), 44. 
T A B LEA U 1

Évolution de la population scolaire en Mauricie entre 1852 et $1900^{1}$

\begin{tabular}{l|c|c|c|c}
\hline \multicolumn{1}{c|}{ Année } & $\begin{array}{c}\text { Population } \\
\text { totale }\end{array}$ & $\begin{array}{c}\text { Nombre } \\
\text { d'enfants âgés } \\
\text { de 5 à 16 ans }\end{array}$ & $\begin{array}{c}\text { Nombre } \\
\text { d'élèves }\end{array}$ & $\begin{array}{c}\text { \% des élèves } \\
\text { par rapport à } \\
\text { la population totale }\end{array}$ \\
\hline $1852-1853^{2}$ & 41458 & 13131 & 4689 & 11 \\
$1862^{3}$ & 51956 & n.d. & 7079 & 14 \\
$1872-1873^{4}$ & 55794 & n.d. & 9706 & 17 \\
$1892-1893^{5}$ & 68197 & 15066 & 11976 & 18 \\
$1900-1901^{6}$ & 77139 & 17747 & 15443 & 20 \\
\hline
\end{tabular}

I. Les données de ce tableau proviennent des rapports annuels du surintendant, exception faite de celles consacrées à la population totale, qui sont tirées des recensements décennaux faits par le gouvernement du Canada. Le choix des années est déterminé par le premier rapport des inspecteurs en 1852-1853 et se poursuit à tous les dix ans sauf pour la dernière décennie de notre période. L'année I882-I883 n'a pu être retenue étant donné que les tableaux statistiques de l'inspecteur Lefebvre, qui vont de 1877 à 1891, intègrent huit localités du comté de Portneuf. Précisons que le nombre des élèves comprend les écoliers protestants.

2. RSIP I852-1853. Voir appendice du onzième volume des journaux de l'Assemblée législative de la Province du Canada Session 1852-53 : Rapport de Petrus Hubert, inspecteur d'écoles, Yamachiche, 5 mars I853, 52.

3. RSIP I862. Pendant quelques années, les rapports annuels correspondent à l'année civile et non à l'année scolaire qui va du ler juillet au 30 juin. Les données sont tirées du Grand tableau statistique préparé d'après les rapports des inspecteurs, des commissaires, etc., 42-43. Des 7189 élèves nous soustrayons les 110 élèves du collège de Trois-Rivières.

4. RSIP 1872-1873, Récapitulation des grands tableaux statistiques préparés d’après les rapports des inspecteurs d'école, etc., 194-195. Nous soustrayons les 160 élèves du collège de Trois-Rivières.

5. RSIP 1892-1893, Statistique générale concernant les élèves [...], I84-185 et Récapitulation, I74-I77.

6. RSIP 1900-1901, Statistiques se rapportant aux enfants d'âge à fréquenter les écoles [...], 234-237 et Récapitulation des statistiques scolaires de la Province, 246-249; des 15702 élèves inscrits à ce tableau, nous soustrayons les 259 élèves du collège de Trois-Rivières.

la suite, des baisses de population scolaire reliées à ce phénomène. On constate, certes, de brèves reprises ici et là, mais insuffisantes pour contrer le ralentissement général. Ainsi, dans son rapport de 1877-1878, Ambroise Tétreault relie la perte de deux cents élèves à la «crise monétaire», qui provoque la fermeture de trois scieries et l'exode de plus de deux cents familles de la circonscription de Maskinongé2 ${ }^{2}$. Il n'y a pas cependant que l'émigration qui provoque un fléchissement de l'affluence dans les écoles. La pauvreté engendrée par le manque de travail peut empêcher l'inscription d'un certain nombre d'enfants ${ }^{23}$. Toutefois, qui dit ralentissement ne dit pas

22. RSIP 1877-1878, Rapport de Ambroise Tétreault, 7.

23. Ian E. Davey, "Trends in Female School Attendance in Mid-Nineteenth-Century Ontario ", dans Ruby Heap and Alison Prentice, dir., Gender and Education in Ontario : An Historical Reader (Toronto, Canadian Scholars' Press, 1991), 5-6; et J. I. Little, Crofters and Habitants. Settler Society, Economy, and Culture in a Quebec Township, 1848-1881, op. cit., 237. 
recul. Le tableau 1 montre que l'augmentation du nombre des élèves reste supérieure à celle de la population. En dépit de la conjoncture, l'effort de scolarisation a donc été poursuivi. Dans la dernière décennie, l'amélioration de la situation économique se reflète par une fréquentation accrue.

Par ailleurs, la distinction des élèves selon le sexe apparaît dans le Rapport du surintendant de l'Instruction publique de 1856 et réapparaît chaque année par la suite à compter de 1873-1874. L'examen des données montre que la répartition des garçons et des filles reste semblable tout au long de la période, fléchissant légèrement de $52 \%$ pour les premiers en 1856 à $48 \%$ en 1900-1901. Sur ce point, la Mauricie ne diffère pas tellement du profil provincial. En 1856, pour l'ensemble du Québec, les élèves des deux sexes fréquentent l'école dans des proportions semblables, tandis qu'en 19001901 , ils se répartissent à raison de $49 \%$ pour les garçons et $51 \%$ pour les filles ${ }^{24}$. Durant la même période, les écoles publiques ontariennes et celles des États de la Nouvelle-Angleterre offrent un portrait similaire ${ }^{25}$.

Le profil de la population scolaire se modifie lorsque les élèves atteignent l'âge de 14 ans. En Mauricie, un plus grand nombre de filles suivent les programmes d'études dits "supérieurs», avec $54 \%$ en leur faveur en 1856 et $68 \%$ en 1900-1901. Nous reviendrons plus loin sur cette catégorie d'élèves. Comparativement à $57 \%$ pour l'ensemble de la province de Québec en 1900-1901, le ratio des écolières de la Mauricie âgées de 14 ans et plus est nettement supérieur. En élargissant la comparaison, on constate que le pourcentage plus élevé d'écolières au-dessus de 13 ans trouve un écho dans les «high schools» publics américains ${ }^{26}$ ainsi qu'en France ${ }^{27}$. Toutefois, dans les «grammar schools» de l'Ontario, l'égalité prévaut en $1890^{28}$.

24. RSIP 1856, 29 et 41; et RSIP 1900-1901, Statistique se rapportant aux enfants d'âge à fréquenter les écoles et aux élèves inscrits aux registres des écoles, 234-237.

25. Ian E. Davey, "Trends in Female School Attendance in Mid-Nineteenth-Century Ontario », op. cit.; Susan E. Houston et Alison Prentice, Schooling and Scholars in Nineteenth-Century Ontario, 202; David Tyack et Elisabeth Hansot, Learning Together. A History of Coeducation in American Public School, 46.

26. John H. Rury, Education and Women's Work. Female Schooling and the Division of Labor in Urban American, 1870-1930 (New York, State University of New York, 1991), 17. Les écolières représentent $53 \%$ de la population scolaire des high schools américains en 1872 et $57 \%$ en 1900.

27. J.-P. Briand, J.-M. Chapoulie, F. Huguet, J.-N. Luc, A. Prost, L'enseignement primaire et ses extensions. Annuaire statistique, $19^{e}-20^{e}$ siècles (Paris, Économica et Service d'histoire de l'éducation, 1987), 138. À en juger par les données de 1924-1925, les écoles comptent $54 \%$ d'écolières de plus de 13 ans comparativement aux garçons du même âge. Les données concernant le sexe des élèves de 14 ans et plus ne sont pas disponibles avant cette date.

28. R. D. Gidney et W. P. J. Millar, Inventing Secondary Education. The Rise of the High School in Nineteenth-Century Ontario (Montréal, McGill-Queen’s University Press, 1990), 329. 


\section{2 - Fréquentation selon la catégorie d'école}

a - Les élèves des écoles élémentaires

Il est malheureusement impossible de répartir avec précision les élèves des écoles élémentaires selon leur âge. Dans les statistiques du département de l'Instruction publique (DIP), la population scolaire est en effet répartie en quatre catégories d'âge : les élèves qui ont 5 et 6 ans, ceux qui ont entre 7 et 13 ans, ceux de 14 et 15 ans et ceux de 16 ans et plus. La catégorie des 7-13 ans prédomine. En 1856, elle rassemblait $77 \%$ de l'ensemble des élèves; en 1900-1901, elle retenait encore $71 \%$. Faute de pouvoir subdiviser cette catégorie, nous devrons lui donner la part du lion dans les pages qui suivent.

Bien que l'on impose aux contribuables une rétribution mensuelle pour les activités scolaires, et obligatoirement pour les enfants âgés de 7 à 13 ans qu'ils aillent ou non en classe, leur présence à l'école ne se généralise pas rapidement, comme l'indique le tableau 2. Si le gouvernement voit dans cet impôt un moyen de rendre l'école obligatoire, la majorité des parents, en dépit du

TA B LE A U 2

Proportion des enfants entre 7 et 13 ans fréquentant l'école,
sur la totalité des enfants du même âge, dans chaque comté
et dans la ville de Trois-Rivières (en pourcentage)

\begin{tabular}{l|c|c|c|c}
\hline \multirow{2}{*}{} & \multicolumn{4}{|c}{ Année $^{1}$} \\
\cline { 2 - 5 } & $\mathbf{1 8 5 6}$ & $\mathbf{1 8 8 4 - 1 8 8 5}$ & $\mathbf{1 8 9 3 - 1 8 9 4}$ & $\mathbf{1 9 0 0 - 1 9 0 1}$ \\
\hline Champlain & 59 & 81 & 91 & 93 \\
Maskinongé & 66 & 83 & 83 & 100 \\
Saint-Maurice & 82 & 79 & 81 & 97 \\
Ville de Trois-Rivières & 62 & n.d. & 81 & 82 \\
\hline Total & 66 & 81 & 86 & 94 \\
\hline
\end{tabular}

I. Le choix des années est basé sur les informations disponibles dans les RSIP. Nous n'avons pu éviter l'écart entre 1856 et 1884. Par contre, nous aurions aimé utiliser les chiffres de l'année 1892-1893, année mitoyenne entre 1884 et 1900, mais les renseignements étaient incomplets.

Sources: 1856: Rapport du recensement, 40-4I. I884-I885: RSIP 1884-1885. Le tableau Écoles communes Sommaire des statistiques fournies par les secrétaires-trésoriers, Récapitulation générale, 282-285, ne comprend pas Trois-Rivières, le recensement des enfants n'ayant pas été fourni. 1893-1894 : RSIP I893-1894, d'après les trois tableaux suivants : Récapitulation; Statistique générale du coût de l'enseignement et du nombre d'enfants de 5 à 16 ans dans la province, 170-173; Statistique générale des écoles élémentaires, 176-179; Statistique générale des écoles modèles et des académies, 184-185. 1900-1901 : RSIP 1900-1901, Statistique se rapportant aux enfants d'âge à fréquenter les écoles et aux élèves inscrits aux registres des écoles, 234-237. Nous excluons les 259 élèves du collège de Trois-Rivières. 
fait qu'ils paient cette taxe, ne considèrent pas l'école comme une priorité et les corvées domestiques ont très souvent préséance sur les travaux scolaires. La pauvreté et l'éloignement de l'école empêchent également la fréquentation d'un certain nombre, faute de vêtements convenables ou de moyens de transport. En 1856, on voit que le ratio se situait aux deux tiers; et il était plus élevé dans la circonscription de Saint-Maurice. Il est difficile d'expliquer la forte avance de cette circonscription, la moins bien nantie des trois. L'explication la plus plausible pourrait venir du fait qu'au milieu du $\mathrm{xIx}^{\mathrm{e}}$ siècle, Saint-Maurice avait une population plus faible que celle des deux autres circonscriptions et concentrée principalement dans les villages du sud, notamment à Yamachiche, ce qui permettait d'ériger des écoles plus accessibles aux enfants ${ }^{29}$. Trente ans plus tard, le rattrapage est complété : $80 \%$ des enfants dans chacune des trois circonscriptions fréquentent désormais l'école. Par la suite, cette tendance s'accentue : au tournant du siècle, le ratio moyen dépasse largement les $90 \%$, à l'exception toutefois de Trois-Rivières.

Il peut sembler étonnant que, parmi les 1532 enfants de 7 à 13 ans recensés à Trois-Rivières en 1900-1901, seulement $82 \%$ fréquentent l'école. Quatre ans auparavant, ce ratio était de $75 \%$. Quelques éléments peuvent expliquer cette situation. Il y a d'abord le fait que Trois-Rivières entame son industrialisation, ce qui fait augmenter sa population de manière significative et ce qui suscite à court terme une pénurie de salles de classe. Les procès-verbaux de la corporation scolaire indiquent d'ailleurs que les commissaires prévoient, en avril 1898, réaménager une résidence à proximité de l'école des frères du quartier Sainte-Ursule afin d'y installer trois ou quatre classes. Il est aussi question, en juillet 1900, de reconstruire l'école de garçons du quartier Notre-Dame, trop petite ${ }^{30}$. L'année suivante, les commissaires font agrandir une école de filles dans le quartier Saint-Louis. Ce manque d'espace dans les établissements trifluviens se reflète dans la fréquentation scolaire non seulement des enfants âgés entre 7 et 13 ans mais aussi des plus jeunes. Ainsi, en 1900-1901, il n'y a que $51 \%$ des enfants âgés de 5 et 6 ans dans les écoles de Trois-Rivières alors que dans les circonscriptions de Maskinongé et de Saint-Maurice, les enfants de cet âge

29. Yamachiche comptait déjà onze écoles en 1832 et en 1853, elle sera la première paroisse rurale de la Mauricie à accueillir au village deux communautés religieuses pour diriger des écoles séparées selon le sexe, à l'exemple de Trois-Rivières.

30. Procès-verbaux de la corporation de Trois-Rivières, délibérations en date du 5 juillet 1900. Le secrétaire note que seuls 92 des 200 enfants du quartier Notre-Dame ont été admis à l'école. Les commissaires décident de faire un emprunt de $6000 \$$, afin d'installer l'école des frères dans un nouveau bâtiment, projet qui sera reporté d’une année. 
vont en classe dans des proportions de $82 \%$ et de $97 \%$ respectivement; dans Champlain, tous les enfants de cette catégorie sont inscrits à l'école.

Par ailleurs, des tentatives ont été faites pour encourager la poursuite des études au-delà de 13 ans, mais avec un succès mitigé. Dès que cesse l'obligation de payer la rétribution mensuelle, la plupart des parents retirent leurs enfants de l'école. Lorsque les élèves quittent l'école, après l'avoir fréquentée pendant six ou sept ans, une forte proportion d'entre eux, comme l'illustre le tableau 3, n'ont achevé que les deux premières années d'un cours qui en compte quatre. En 1900-1901, la concentration des élèves dans les deux premières années s'affirme davantage dans Champlain et Saint-Maurice, tandis que dans Maskinongé, les écoliers prolongent leurs

TABLEAU 3

Fréquentation des élèves au cours élémentaire par degrés d'enseignement pour les années 1892-1893 et 1900-1901'

\begin{tabular}{l|r|r|r|r|r}
\hline \multicolumn{7}{c}{$\mathbf{I} 89 \mathbf{2}$ - I 893} \\
\hline \multicolumn{1}{c|}{ Comté ou ville } & $\mathbf{1}^{\text {ère }}$ année & $\mathbf{2}^{\mathbf{e}}$ année & $\mathbf{3}^{\mathbf{e}}$ année & $\mathbf{4}^{\mathbf{e}}$ année & \multicolumn{1}{c}{ Total } \\
\hline Champlain & $2101(50 \%)$ & $1181(28 \%)$ & $797(19 \%)$ & $91(2 \%)$ & $4178(100 \%)$ \\
Maskinongé & $560(26 \%)$ & $996(47 \%)$ & $404(19 \%)$ & $168(8 \%)$ & $2128(100 \%)$ \\
$\begin{array}{l}\text { Saint-Maurice et } \\
\text { ville de Trois-Rivières }\end{array}$ & $773(30 \%)$ & $1155(45 \%)$ & $461(18 \%)$ & $161(6 \%)$ & $2550(100 \%)$ \\
\hline Province de Québec & $75612(40 \%)$ & $52455(28 \%)$ & $36851(20 \%)$ & $20772(11 \%)$ & $185690(100 \%)$ \\
\hline
\end{tabular}

$|900-190|$

\begin{tabular}{l|r|r|r|r|r}
\hline \multicolumn{1}{c|}{ Comté ou ville } & $\mathbf{1}^{\text {ère }}$ année & $\mathbf{2}^{\mathbf{e}}$ année & $\mathbf{3}^{\mathbf{e}}$ année & $\mathbf{4}^{\mathbf{e}}$ année & \multicolumn{1}{c}{ Total } \\
\hline Champlain & $3408(55 \%)$ & $1845(30 \%)$ & $733(12 \%)$ & $152(2 \%)$ & $6138(100 \%)$ \\
Maskinongé & $1267(51 \%)$ & $741(30 \%)$ & $354(14 \%)$ & $112(5 \%)$ & $2474(100 \%)$ \\
Saint-Maurice & $1469(64 \%)$ & $571(25 \%)$ & $233(10 \%)$ & $34(1 \%)$ & $2307(100 \%)$ \\
Trois-Rivières & $309(46 \%)$ & $193(29 \%)$ & $102(15 \%)$ & $62(9 \%)$ & $666(100 \%)$ \\
\hline Province de Québec & $80139(46 \%)$ & $50753(29 \%)$ & $30790(18 \%)$ & $11591(7 \%)$ & $173273(100 \%)$ \\
\hline
\end{tabular}

I. Ces statistiques ne dénombrent que les élèves inscrits dans les écoles dites élémentaires à l'exclusion de ceux qui suivent ce programme d'études dans les écoles modèles et académiques. Le choix des années correspond à la première présentation de cette information.

Sources: 1892-1893, Statistique générale concernant les élèves, d’après les bulletins des inspecteurs, 184-185. Les pourcentages ont été arrondis. RSIP 1900-1901, Écoles élémentaires catholiques, Statistique se rapportant au cours d'études suivi par les élèves, 190-192 et Écoles modèles, Statistique se rapportant [...], 202-204 et Écoles académiques, Statistique se rapportant [...], $210-212$. 
études. Pour leur part, les élèves trifluviens complètent leurs études élémentaires en une proportion un peu plus grande que ceux du monde rural. Comparativement à l'ensemble de la province de Québec, en 19001901, force est de constater qu'au terme de leur séjour à l'école, les enfants disséminés dans la campagne mauricienne sont un peu moins instruits que la moyenne québécoise. En 1913, l'inachèvement de la formation persiste comme le constate l'inspecteur général des écoles, Charles-Joseph Magnan, qui indique que peu d'élèves terminent le cours élémentaire, quittant plutôt vers «l'âge de 13 ou 14 ans, avant d'avoir atteint au moins la quatrième année, couronnement du cours élémentaire ${ }^{31}$ ».

\section{b - Les élèves des écoles modèles et académiques}

Il est nécessaire de préciser, de prime abord, que seule une minorité d'élèves, dans les écoles modèles et académiques, sont inscrits dans les cours modèle et académique. La grande majorité des élèves de ces écoles suivent le cours élémentaire. C'est ainsi qu'en 1900-1901, on compte 4018 élèves fréquentant ces écoles; et parmi eux, seulement 784, soit 19,5\%, sont de niveau "supérieur». Durant le dernier quart du XIX ${ }^{\mathrm{e}}$ siècle, la proportion des élèves de ce niveau dans les trois circonscriptions de la Mauricie reste pratiquement inchangée, oscillant entre $5 \%$ et $6 \%$ de la population scolaire totale. La constance de ce faible pourcentage indique une grande précarité. Les écoles modèles du monde villageois recrutent difficilement le minimum d'élèves requis et, surtout, conservent avec peine le statut "supérieur» de l'école garantissant la subvention gouvernementale à la corporation. Plusieurs écoles modèles sont menacées, à un moment ou à un autre, de perdre leur statut si le minimum de 6 élèves étudiant toutes les matières du cours modèle n'est pas atteint. La chose se produit parfois à la suite de l'implantation d'un pensionnat de filles au village, car les religieuses enseignantes offrent généralement le cours modèle. L’ancienne école mixte se transforme alors en une école de garçons qui compte moins d'élèves. C'est dans de telles circonstances que l'école modèle de garçons du village de Saint-Maurice perd son allocation en 1893-1894². L’année

31. Charles-Joseph Magnan, «À propos de fréquentation scolaire» dans Au service de mon pays, Discours et conférences (Québec, Dussault et Proulx, 1917), 495.

32. ANQ, Correspondance reçue de Saint-Maurice, document $n^{\circ} 2166,1894$ : réponse du surintendant en date du 17 novembre 1894. Cette lettre est tirée de la correspondance reçue au département de l'Instruction publique entre 1850 et 1900 conservée aux Archives nationales du Québec (ANQ), dans le fonds E 13, Fonds du ministère de l'Éducation, localisation 1A18-2103B à $1 \mathrm{~A} 24-2302 \mathrm{~A}$, articles numérotés 311 (1850) à 935 (1900). Pour éviter la répétition, nous omettrons la localisation. 
suivante, 8 élèves suivront le programme, ce nombre restant stable jusqu'en 1901. Pendant ce temps, le couvent du village offre le cours modèle à 6 filles (1896-1897), ce nombre grimpant à 15 à la fin du siècle. Les pensionnats ne sont cependant pas à l'abri d'un manque d'effectifs et perdent, à l'occasion, leur subvention comme c'est le cas, en 1888 , du couvent de Saint-Paulin où seulement 4 écolières suivent, en partie, le programme du cours modèle $e^{33}$.

Il n'empêche que le nombre des élèves inscrits au cours supérieur à travers la Mauricie est passé de 348 en 1856 à 808 en 1900-1901. Et comme le montre le tableau 4, la proportion des élèves de 14 ans et plus est plus grande à Trois-Rivières en 1900-1901, atteignant $13 \%$ contre $5 \%$ dans les autres circonscriptions.

TABLEA U 4

Population scolaire âgée de 14 ans et plus en 1900-1901

\begin{tabular}{|c|c|c|c|c|c|}
\hline Comté ou ville & $\begin{array}{l}\text { Comté de } \\
\text { Champlain }\end{array}$ & $\begin{array}{l}\text { Comté de } \\
\text { Maskinongé }\end{array}$ & $\begin{array}{c}\text { Comté de } \\
\text { Saint-Maurice }\end{array}$ & \begin{tabular}{|c|} 
Ville de \\
Trois-Rivières
\end{tabular} & Total \\
\hline $\begin{array}{l}\text { Écoliers } \\
\text { de } 14-15 \text { ans }\end{array}$ & 106 & 54 & 20 & 92 & 272 \\
\hline $\begin{array}{l}\text { Écoliers } \\
\text { de } 16 \text { ans et plus }\end{array}$ & 12 & 4 & 2 & 0 & 18 \\
\hline $\begin{array}{l}\text { Écolières } \\
\text { de } 14-15 \text { ans }\end{array}$ & 164 & 83 & 97 & 100 & 444 \\
\hline $\begin{array}{l}\text { Écolières } \\
\text { de } 16 \text { ans et plus }\end{array}$ & 72 & 20 & 36 & 36 & 164 \\
\hline Total des 14 et plus & 354 & 161 & 155 & 228 & $898^{1}$ \\
\hline $\begin{array}{l}\text { Population } \\
\text { scolaire totale }\end{array}$ & 7505 & 3224 & 3008 & 1706 & 15443 \\
\hline$\%$ des 14 ans et plus & 5 & 5 & 5 & 13 & 6 \\
\hline
\end{tabular}

I. Parmi ces 898 élèves, il s'en trouve 90 qui poursuivent leurs études élémentaires. Les 808 élèves étudiant le programme des cours modèle et académique se répartissent ainsi : 784 dans les écoles dites supérieures et 24 dans les écoles élémentaires, Résumé des collèges classiques, des académies et des écoles modèles catholiques subventionnées, 258-29I et Statistique se rapportant au cours d'études suivant les élèves [des] Écoles élémentaires catholiques, 190-192.

Source : Rapport du surintendant de l'Instruction publique pour l'année 1900-1901, Statistique se rapportant aux enfants d’âge à fréquenter les écoles et aux élèves inscrits aux registres des écoles, 234-237.

33. ANQ, Correspondance reçue de Saint-Paulin, document $\mathrm{n}^{\circ} 371,1888$ : réponse du surintendant en date du 16 mai 1888. 
Le même tableau expose la diversité des parcours des garçons et des filles. Dans le monde villageois, à peine une vingtaine de garçons de plus de 15 ans poursuivent leurs études dans le système d'instruction publique, comparativement à 128 filles. À Trois-Rivières, parmi les élèves de 14-15 ans, il y a presque autant de garçons que de filles. Par contre, au-delà de 15 ans, aucun garçon ne fréquente une école publique, tandis que 36 jeunes filles de cet âge y sont inscrites, plusieurs étant sans doute attirées par l'enseignement, le seul métier accessible pour celles qui sont instruites. Quant aux filles qui ne se dirigent pas vers l'enseignement, elles perçoivent la prolongation des études comme une amélioration personnelle en attente du mariage ${ }^{34}$.

Au total, la population du réseau d'écoles publiques est constituée d'une forte majorité d'enfants âgés entre 5 et 13 ans. La fréquentation scolaire devient, peu à peu, un passage incontournable pour eux et pour quelquesuns de leurs aînés. Il ne fait pas de doute que la multiplication des écoles dans les trois circonscriptions est responsable de ce résultat. Cependant, vue sous l'angle de l'assiduité, la situation s'assombrit quelque peu.

\section{B - L'assiduité}

L'assiduité en classe, c'est-à-dire la présence régulière des élèves, apparaît comme un objectif bien difficile à atteindre. Les corporations scolaires essaient de trouver des solutions afin de contrer les absences, tandis que plusieurs curés, du haut de la chaire, exhortent les parents à envoyer régulièrement leurs enfants à l'école. Pour leur part, au terme de leurs visites, les inspecteurs ne cessent de s'étonner de la faible assistance dans les classes. Tout comme Petrus Hubert, qui affirme au début des années 1870 «que le progrès [des écoles] laisse généralement à désirer surtout à la campagne, sous le rapport de l'assiduité des élèves ${ }^{35}$ ", les inspecteurs reviennent constamment sur ce sujet dans leurs rapports par la suite. Ils commentent et analysent cet aspect de la vie scolaire en cherchant des responsables et en énumérant les obstacles entraînant l'absentéisme des écoliers.

Le tableau 5 expose les taux d'assistance moyenne entre 1873 et 1901 , aussi bien dans les écoles élémentaires que dans les écoles modèles et académiques. Dans ce dernier cas, comme le dénombrement des statistiques gouvernementales ne tient pas compte du niveau d'études des élèves, il est

34. Denise Lemieux et Lucie Mercier, Les femmes au tournant du siècle, 1880-1940. Âges de la vie, maternité et quotidien (Québec, Institut québécois de recherche sur la culture, 1989), 85, et Collectif Clio, L'histoire des femmes au Québec depuis quatre siècles (Montréal, Le Jour, éditeur, 1992), 197.

35. RSIP 1872-1873, Rapport de Petrus Hubert, 99. 
TABLEAU 5

Assistance moyenne ${ }^{1}$ dans les écoles élémentaires, modèles et académiques de la Mauricie entre 1873 et $1901^{2}$

| 873 - | 874

\begin{tabular}{l|c|c}
\hline District ou comté & $\begin{array}{c}\text { Écoles } \\
\text { élémentaires (\%) }\end{array}$ & $\begin{array}{c}\text { Écoles modèles et } \\
\text { académiques (\%) }\end{array}$ \\
\hline $\begin{array}{l}\text { Comtés de Champlain, de Maskinongé } \\
\text { et de Saint-Maurice réunis } \\
\text { Taux moyen pour la Mauricie }\end{array}$ & 70 & 78 \\
\hline Province de Québec & 73 & 84 \\
\hline
\end{tabular}

| 877 - | 878

\begin{tabular}{l|c|c}
\hline District ou comté & $\begin{array}{c}\text { Écoles } \\
\text { élémentaires (\%) }\end{array}$ & $\begin{array}{c}\text { Écoles modèles et } \\
\text { académiques (\%) }\end{array}$ \\
\hline Champlain et partie de Portneuf & 64 & 82 \\
\hline Maskinongé et Saint-Maurice & 65 & 88 \\
\hline Taux moyen pour la Mauricie & 64,5 & 85 \\
\hline Province de Québec & 73 & 84 \\
\hline
\end{tabular}

| 887 - | 888

\begin{tabular}{l|c|c}
\hline District ou comté & $\begin{array}{c}\text { Écoles } \\
\text { élémentaires (\%) }\end{array}$ & $\begin{array}{c}\text { Écoles modèles et } \\
\text { académiques (\%) }\end{array}$ \\
\hline Champlain et partie de Portneuf & 64 & 75 \\
\hline Maskinongé et Saint-Maurice & 70 & 81 \\
\hline Taux moyen pour la Mauricie & 67 & 78 \\
\hline Province de Québec & 72 & 81 \\
\hline
\end{tabular}

| 893 - | 894

\begin{tabular}{l|c|c}
\hline District ou comté & $\begin{array}{c}\text { Écoles } \\
\text { élémentaires (\%) }\end{array}$ & $\begin{array}{c}\text { Écoles modèles et } \\
\text { académiques (\%) }\end{array}$ \\
\hline Champlain & 66 & 84 \\
\hline Maskinongé & 69 & 86 \\
\hline Saint-Maurice & 72 & 81 \\
\hline Taux moyen pour la Mauricie & 69 & 84 \\
\hline Province de Québec & 71 & 84 \\
\hline
\end{tabular}


TABLEAU 5 (suite)

\section{Assistance moyenne ${ }^{1}$ dans les écoles élémentaires, modèles et académiques de la Mauricie entre 1873 et $1901^{2}$}

\begin{tabular}{l|c|c}
\hline \multicolumn{3}{c}{1900 - 190 I } \\
\hline District ou comté & $\begin{array}{c}\text { Écoles } \\
\text { élémentaires (\%) }\end{array}$ & $\begin{array}{c}\text { Écoles modèles et } \\
\text { académiques (\%) }\end{array}$ \\
\hline Champlain & 64 & 81 \\
\hline Maskinongé & 68 & 82 \\
\hline Saint-Maurice & 67 & 86 \\
\hline Taux moyen pour la Mauricie & 66,3 & 83 \\
\hline Province de Québec & 68 & 82 \\
\hline
\end{tabular}

I. L'assistance moyenne est une donnée des rapports statistiques qui mesure la présence effective des élèves en classe. Elle est transmise par l'enseignant à l'inspecteur des écoles sous forme de chiffres. Pour établir les taux de ce tableau, nous avons divisé la colonne totalisant l'Assistance moyenne des élèves par le Total des élèves inscrits aux écoles élémentaires et multiplié le résultat par 100; puis nous avons répété l'exercice pour ceux inscrits dans les écoles modèles et académiques.

2. Le choix des années s'explique ainsi : 1873-1874, première mention de la présence moyenne en classe; 1877-1878, nouvelle répartition des données correspondant aux deux districts d'inspection de la Mauricie, soit celui du comté Champlain et partie de Portneuf et celui des comtés Maskinongé et de Saint-Maurice; 1887-1888, la situation dix ans plus tard; 1893-1894, la notion de district cède la place aux comtés et constitue une période mitoyenne avant la fin de la période, 1900-1901. Les données du rapport du surintendant ne sont pas ventilées par comté avant I891-1892.

Source: RSIP 1873-1874. D'après le tableau Récapitulation. Grand tableau statistique préparé d'après les rapports des inspecteurs, des commissaires [...], 26-27. RSIP I877-1878. Idem, I08-II5. RSIP I887-I888. D'après le tableau Statistique générale des écoles sous contrôle des corporations scolaires, 194-199. RSIP 1893-1894. D'après les tableaux Statistique générale des écoles élémentaires [...], 176-179; Statistique générale des écoles modèles et académiques [...], I80-183. RSIP 1900-1901. D’après les tableaux Écoles élémentaires catholiques, I86-I87; Écoles modèles, 198-20I ; Académies catholiques, 206-209.

impossible de préciser s'il s'agit d'élèves inscrits à l'élémentaire, au cours modèle ou au cours académique. Rappelons que les écoles dites "supérieures » sont composées en grande partie d'élèves du cours élémentaire. Ajoutons que ces données proviennent des registres tenus par les titulaires de classe, qui doivent inscrire chaque jour les présences. Lors de sa visite, l'inspecteur cueillera cette information et l'insérera dans son bilan annuel.

Au milieu des années 1870, on évalue à $70 \%$ l'assiduité moyenne des élèves du niveau élémentaire et à $78 \%$ celle des écoliers inscrits dans les écoles modèles ou académiques et dans les institutions indépendantes. Par la suite, le taux de l'assistance moyenne baisse quelque peu dans les écoles élémentaires, tandis qu'il s'élève dans les écoles supérieures.

Divers facteurs peuvent expliquer la baisse de ce taux sous le seuil de $70 \%$ au cours des années 1873-1900. D’abord, une manière plus précise de calculer 
cette moyenne a pu avoir été adoptée. La méthode de l'inspecteur Petrus Hubert (1852-1876) pour évaluer l'assiduité des élèves reste inconnue. Son successeur David Lefebvre, constatant que la plupart des institutrices ne donnent qu'un nombre approximatif, leur apprend «à calculer, mois par mois, l'assistance moyenne d'une manière exacte», mais l'inspecteur n'élabore pas davantage sur la formule employée ${ }^{36}$.

Ensuite, les fonctionnaires du département de l'Instruction publique, lors de leurs tournées, ne manquent pas d'observer et d'analyser le retard de la Mauricie. Les inspecteurs rendent d'abord le personnel enseignant responsable du manque d'assiduité de leurs élèves. Ambroise Tétreault souligne que «dans toutes les bonnes écoles, l'assistance est généralement satisfaisante et atteint même quelquefois jusqu'à quatre-vingt-dix par cent; tandis qu'à dix pas plus loin, dans la même concession, l'école est déserte ${ }^{37}$ ». Son confrère David Lefebvre en fait presque un leitmotiv, tant il revient fréquemment sur le lien entre une école bien dirigée et une assistance régulière.

Il est vrai que la très grande majorité des instituteurs et institutrices de la Mauricie n'ont pas de véritable formation pédagogique. La loi de 1846 oblige certes les instituteurs à détenir l'un des trois brevets d'enseignement dispensés par les bureaux d'examinateurs et qui déterminent le niveau d'enseignement : élémentaire, modèle ou académique. Les candidats à l'enseignement disposent à partir de 1853 d'un bureau d'examinateurs, situé à Trois-Rivières. Cependant, la région ne bénéficie pas d'école normale sur son territoire. Si bien qu'en 1900-1901, parmi les 247 membres du personnel enseignant, $98 \%$ ont opté pour le brevet émis par un bureau d'examinateurs plutôt que d'effectuer des études d'une ou deux années dans une école normale. Dans l'ensemble de la province la proportion du personnel à avoir fait le même choix est de $83 \%^{38}$. Le brevet d'enseignement émis par un bureau d'examinateurs régional, en principe, devrait posséder la même valeur qu’un brevet émis par une école normale. En pratique, il en va tout autrement car chaque bureau, avant la création du Bureau central, en 1898, détermine les questions soumises aux candidats, d'où les multiples reproches de "trop grande facilité» qui sont formulés à leur endroit en de nombreuses occasions ${ }^{39}$.

36. RSIP 1882-1883, Rapport de David Lefebvre, 59.

37. RSIP 1878-1879, Rapport de Ambroise Tétreault, 114.

38. Jocelyne Murray, op. cit., 403.

39. RSIP 1856, 11 et RSIP 1896-1897, viii. Voir aussi J.-P. Charland, L'entreprise éducative au Québec, op. cit., 310-311. 
Les conditions socio-économiques des contribuables ont elles aussi été évoquées par les inspecteurs. Très souvent, les enfants s'absentent de l'école pour accomplir des tâches domestiques ou encore pour travailler. L'inspecteur J. O. Goulet remarque «que les parents requièrent les services de leurs enfants bien jeunes. Ainsi, pendant le temps des semences, des récoltes, et pour un rien, on garde les enfants chez $\operatorname{soi}^{40}$ ». Le calendrier scolaire entre en conflit avec les travaux saisonniers. Les aînés accompagnent les parents aux champs, tandis que ceux et celles qui sont assez vieux pour surveiller les tout-petits restent à la maison ${ }^{41}$. Plusieurs élèves manquent ainsi le début de l'année scolaire et quelques-uns semblent incapables de rattraper ce retard. Pour sa part, le curé de Cap-de-la-Madeleine note que l'entreprise d'allumettes de son village compromet l'assiduité des élèves à l'école ${ }^{42}$. Les «maladies épidémiques» retiennent également les écoliers à la maison ${ }^{43}$.

À ces facteurs endémiques, les inspecteurs en ajoutent d'autres, plus matériels. Le délabrement de certaines écoles et l'exiguiité des locaux n'encouragent pas toujours les écoliers à l'assiduité. Un mobilier inadapté à la taille des enfants, composé de longues tables de dix à douze pieds occupées par une dizaine d'élèves assis sur des bancs collectifs, avec ou sans dossiers, et une température trop chaude ou trop froide créent des conditions où «il n'y a pas d'assistance, $[. .$.$] les enfants fuient l'école comme$ l'oiseau fuit la cage ${ }^{44} »$. Dans les régions nouvellement peuplées, vivant principalement de la forêt et de la sidérurgie, l'implantation et l'organisation matérielle des écoles posaient de graves problèmes pécuniaires. Les corporations scolaires de Saint-Élie, de Saint-Mathieu ou de Saint-Sévère, dans la circonscription de Saint-Maurice, et celles de Fermont, de SaintAdelphe et de Saint-Jacques-des-Piles, dans celle de Champlain, durent, au tournant du siècle,recevoir une aide financière supplémentaire afin de mieux desservir leur population.

En revanche, le tableau 5 indique une hausse de l'assiduité dans les écoles modèles et académiques, toujours situées dans les villages ou à la ville. La

40. RSIP, 1901-1902, Rapport de J. O. Goulet, 44.

41. ANQ, correspondance reçue de Sainte-Geneviève, document $n^{\circ} 2510,1878$ : lettre du secrétaire-trésorier Robert Trudel en date du 24 septembre.

42. Carmen Rousseau, «Les Oblats et l'encadrement paroissial au Cap-de-la-Madeleine, 19021918 », dans Serge Gagnon et René Hardy, L'Église et le village au Québec, 1850-1930. L'enseignement des Cahiers de prônes (Montréal, Leméac, 1979), 135.

43. RSIP, 1885-1886, Rapport d'Ambroise Tétreault, 95 ; RSIP, 1886-1887, Rapport d'A. Tétreault, 123 ; RSIP, 1901-1902, Rapport de J. O. Goulet, 44.

44. RSIP 1877-1878, Rapport de Ambroise Tétreault, 93. 
proximité de la population, la facilité du parcours et la moins grande mobilisation des petits villageois et des citadins pour accomplir des corvées domestiques favorisent cette présence. Ajoutons que les écoles de village ou de la ville répondent généralement aux exigences des inspecteurs. Par ailleurs, les écoles de village sont considérées comme les meilleures de la corporation, ce qui encourage une assistance régulière. Il n'est donc pas surprenant que les pourcentages d'assiduité montent à $80 \%$ et plus.

D’une manière générale, les taux d'assiduité présentent une assez grande stabilité, se situant grosso modo à $67 \%$ pour le niveau élémentaire dispensé dans les "petites écoles» de rang et à $82 \%$ pour les écoles modèles et académiques. Comme l'indique le tableau 5 , la Mauricie affiche une performance un peu moins bonne que la moyenne québécoise. Mais en 1900-1901, les pourcentages sont assez semblables à tous les niveaux d'enseignement.

Le problème de l'assiduité préoccupe certainement les commissaires. Ils s'attardent surtout à trouver des solutions. Ainsi, pour contrer les difficultés liées à la rigueur du climat, certains souhaitent allonger la période de vacances "du jour de l'An», quitte à raccourcir d'autant celle de l'été ou même à fixer

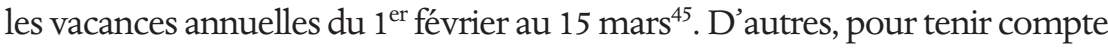
du travail des enfants durant la saison des foins et des récoltes, suggèrent que les vacances soient fixées du $1^{\text {er }}$ août au 15 septembre ${ }^{46}$.

Certaines corporations cherchent, par contre, à tirer profit d'une faible assiduité. En effet, des commissaires, aux prises avec un manque de ressources, sollicitent la permission d'engager une institutrice non brevetée pour les écoles où l'assistance moyenne des élèves n'est pas élevée. Ainsi, le secrétaire-trésorier de la paroisse de Saint-Tite demande l'autorisation d'embaucher une maîtresse non diplômée pour l'école $\mathrm{n}^{\circ} 3$, "peu fréquentée», c'est-à-dire où il n'y a que neuf enfants assidus sur trente-quatre. Selon lui, une «institutrice qui pourrait enseigner les premiers éléments des sciences et le catéchisme pourrait répondre au zèle sinon aux exigences des parents ${ }^{47}$ ».

Lorsqu'il est question du financement de l'instruction publique, nous pouvons affirmer que, pour les corporations scolaires, aucune décision n'est aussi délicate qu'une hausse d'impôt ${ }^{48}$. Par souci d'économie ou par

45. ANQ, Correspondance reçue de Saint-Luc, document $\mathrm{n}^{\circ}$ 1246, 1900 ; de Saint-Jean-desPiles, document $\mathrm{n}^{\circ}$ 1966, 1898; de Saint-Jacques-des-Piles, document $\mathrm{n}^{\circ}$ 1495, 1900 et de SaintNarcisse, document $\mathrm{n}^{\circ} 1817,1900$.

46. ANQ, Correspondance reçue de Saint-Narcisse, document $\mathrm{n}^{\circ}$ 1090, 1892.

47. ANQ, Correspondance reçue de Saint-Tite, document $n^{\circ} 945,1890$ : lettre en date du 22 juillet.

48. Jocelyne Murray, op. cit., chapitre 2, 72-132. 
incompréhension des besoins réels des écoliers et de leurs maîtres, les commissaires laissent trop souvent les écoles de campagne dans un piètre état, tardent à moderniser leur mobilier et n'offrent pas de traitements alléchants à leur personnel. Sur ce plan encore, apparaît une dichotomie entre les écoles de village et les écoles de rang. Il existe au sein des corporations rurales une hiérarchisation des écoles voulant que celle du village soit la meilleure, tant au niveau de l'enseignement que du confort.

L'assistance moyenne demeure, somme toute, relativement basse durant le dernier quart du $\mathrm{XIX}^{\mathrm{e}}$ siècle. Il va de soi que le manque d'assiduité des élèves impose une limite à l'acquisition des connaissances. En 1900-1901, la presque totalité des enfants entre 7 et 13 ans sont inscrits à l'école, mais étant donnée l'irrégularité de leur présence en classe, ils ne peuvent, pour la plupart, suivre en entier le programme scolaire, comme le montre le tableau 3. Ils doivent donc sans cesse reprendre les apprentissages là où ils les avaient laissés.

Il faudra bien des années d'efforts avant que les élèves puissent tous lire, écrire et compter.

\section{IV - UNE SCOLARISATION SUR MESURE}

Les statistiques contenues dans les rapports annuels du surintendant permettent de mesurer à la fois les apprentissages et la qualité de l'enseignement. Entre 1850 et 1891, des progrès ont eu lieu. Ils se mesurent au nombre d'écoliers apprenant à lire, à écrire et à compter, ainsi qu'au nombre de ceux étudiant des matières comme la grammaire ou la géographie. Cette évaluation par matière permet également de vérifier l'efficacité d'un personnel peu au courant des techniques d'enseignement.

\section{A - Le classement des élèves}

Jusqu'aux années 1890, le classement des élèves, dans les rapports du surintendant de l'Instruction publique, s'effectue sur une base individuelle établie selon l'avancement de chacun dans l'étude des diverses matières inscrites au programme en vigueur. Ainsi, il est possible de connaître combien apprennent, par exemple, à lire, à écrire et à compter dans une localité $e^{49}$, dans une circonscription ou dans la province. Ce procédé n'est pas propre au Québec puisqu'il est également employé dans l'évaluation de la

49. Entre 1861 et 1873 , tous les trois ans, les données sont fournies par localité, ce qui permet une lecture très fine de l'évolution des apprentissages. 
population scolaire des écoles ontariennes ${ }^{50}$. Dans ses premières instructions aux inspecteurs (1851) ainsi que dans sa circulaire du 22 juin 1854, le surintendant Jean-Baptiste Meilleur privilégie cette façon de faire. Son successeur, Pierre-Joseph-Olivier Chauveau, l'adopte à son tour en 1856, car il considère que «le progrès réel [...] doit être jugé beaucoup plus d’après le nombre d'enfants qui profitent de ce qu'on leur enseigne que d'après le nombre de ceux qui fréquentent les écoles ${ }^{51}$ ». Par la suite, en dépit de l'existence de programmes d'études qui définissent la progression des élèves, on continue à les dénombrer par matières.

En général, le classement individuel des élèves reste la responsabilité exclusive du personnel enseignant. Aucun examen officiel émanant du département de l'Instruction publique ne sanctionne les études du cours élémentaire et aucune instance locale n'émet de diplôme de fin d'études. Deux fois par année, les élèves sont évalués. En décembre, un examen semestriel a lieu en présence de quelques commissaires, du secrétaire-trésorier et habituellement du curé. En juillet, au terme de l'année scolaire, les commissaires sont tenus de faire passer un examen public - les parents étant conviés à assister - afin d'apprécier les progrès des élèves et le travail du maître. Les résultats sont consignés dans les registres conservés dans l'armoire de la classe. La nature de l'évaluation au sein des corporations, son déroulement et ses conclusions restent inconnus pour l'instant. Par contre, les rapports annuels du surintendant et ceux des inspecteurs d'écoles contiennent maints commentaires et statistiques sur le progrès des élèves et le travail du personnel enseignant. Nous reprenons, à notre tour, ces informations afin de brosser un tableau de la formation acquise par les écoliers entre 1850 et 1900 .

\section{B - L'évaluation par matière}

L'analyse que nous faisons de l'évolution des apprentissages porte sur une sélection de cinq matières. Aux enseignements de base, lecture, écriture et arithmétique, nous ajoutons la géographie et la tenue des livres. La fameuse trilogie, lire-écrire-compter, apparaît comme l'apprentissage obli-

50. R. D. Gidney et W. P. J. Millar, Inventing Secondary Education. The Rise of the High School in Nineteenth-Century (Montréal, McGill-Queen's University Press, 1990, 411 p. Ces historiens utilisent des données semblables pour illustrer les effectifs des écoles ontariennes transmises annuellement dans l'Annual Report of the Normal, Model, Grammar, and Common Schools in Upper Canada [jusqu'en 1875] et Report of the Minister of Education (Ontario) [après 1875].

51. RSIP 1856, 21. 
gatoire de toute école élémentaire. La géographie, matière dont l'enseignement repose sur l'utilisation d'un matériel pédagogique autre que le manuel, l'ardoise ou le papier, sert en même temps d'indice révélateur de la diversité de l'enseignement élémentaire. Elle peut aussi être considérée comme un incitatif à fréquenter l'école car, d'après l'inspecteur Tétreault, c'est une matière appréciée des enfants. Finalement, la tenue des livres est offerte d'abord aux trois niveaux et, par la suite, seulement dans les écoles modèles et académiques. Comme elle apparaît dans les premiers tableaux statistiques, il est possible de suivre sa progression depuis le début.

À part le catéchisme, qui ne fait pas l'objet d'une évaluation officielle de l'inspecteur des écoles, les élèves apprennent d'abord à lire, puis à écrire et à compter. Le tableau 6 illustre comment les élèves se répartissent selon les matières de notre sélection. En 1854, nous constatons la prééminence d'une matière, la lecture. Quatre-vingt-onze pour cent des écoliers apprennent à lire. Dix ans plus tard, cette proportion a régressé à $82 \%$. Les critères d'évaluation ont-ils changé? S'agit-il des effets d'une forte croissance du nombre des élèves sur une organisation encore peu consolidée? Nous ne possédons aucun indice pouvant expliquer la diminution.

Cependant, en 1872-1873, tous les élèves de la Mauricie apprennent à lire. Et par la suite, ce score parfait sera maintenu, ou presque. Désormais, on ne dénombre plus séparément les élèves qui apprennent l'abc de ceux qui font de la lecture courante. On distingue seulement ceux qui lisent bien des autres.

Toutefois, les autres matières de base ne connaissent pas une popularité aussi précoce. En 1872-1873, l'inspecteur Hubert ne déborde pas d'enthousiasme devant ces résultats qu'il attribue à la faible assiduité des écoliers de la campagne et au manque de fournitures scolaires, "deux obstacles» ayant pour effet de "ralentir l'avancement de l'instruction populaire dans presque toutes les localités rurales de mon district d'inspection ${ }^{52}$ ».

Savoir lire n’implique pas nécessairement savoir écrire. Entre 1854 et 1873, un tiers des élèves seulement sont initiés à l'écriture. Puis, en 1882-1883, une proportion beaucoup plus grande apprend à écrire : 86\% des écoliers comparativement à $37 \%$, dix ans plus tôt. David Lefebvre explique ces progrès par le fait que cet apprentissage exige «que chaque élève ait son ardoise, quelque jeune qu'il soit, afin de lui montrer à écrire en même temps qu'on lui apprend à lire ${ }^{53}$ \%. D’après ses observations, ceux qui n'écrivent pas encore 
TAB LEAU 6

Nombre d'élèves par matières d'enseignement

\begin{tabular}{|c|c|c|c|c|c|c|}
\hline Année & Nombre total d'élèves & Lecture $^{1}$ & Écriture $^{2}$ & Arithmétique $^{3}$ & Géographie & Tenue de livres \\
\hline $1854^{4}$ & 4716 & $4309(91 \%)$ & $1721(36 \%)$ & $1394(30 \%)$ & $405(9 \%)$ & $0(0 \%)$ \\
\hline $1863^{5}$ & 7337 & $6037(82 \%)$ & $2299(31 \%)$ & $2192(30 \%)$ & 940 (13\%) & $197(3 \%)$ \\
\hline $1872-1873^{6}$ & 9866 & $9866(100 \%)$ & $3644(37 \%)$ & $3430(35 \%)$ & $1219(12 \%)$ & $510(5 \%)$ \\
\hline $1882-1883^{7}$ & 13372 & $13372(100 \%)$ & $11565(86 \%)$ & $7251(54 \%)$ & $2974(22 \%)$ & $1567(12 \%)$ \\
\hline $1892-1893^{8}$ & 11976 & $11750(98 \%)$ & $10308(86 \%)$ & $9244(77 \%)$ & 3927 (33\%) & n.d. \\
\hline $1898-1899^{9}$ & 14361 & $14361(100 \%)$ & $14361(100 \%)$ & $14361(100 \%)$ & $9913(69 \%)$ & $954(7 \%)$ \\
\hline
\end{tabular}

I. Pour les années situées entre 1854 et 1882, nous additionnons trois colonnes: «Nombre d'élèves lisant depuis Abc jusqu'à la lecture courante», «Nombre des élèves sachant la lecture courante et Nombre d'élèves lisant bien ». En 1882-1883, il y a deux colonnes : «L'Abc et la lecture courante» et «Lisant bien ». En |89|-|892, il n'y a plus qu'une seule rubrique, «Lecture». Nous avons interprété cette rubrique comme regroupant les élèves qui savent bien lire comme ceux qui débutent. Cela vaut pour toutes les matières du tableau.

2. Entre 1854 et 1890, cette rubrique s'intitule «nombre d'élèves écrivant» puis, en |89|-|892, «écriture».

3. Pour les années situées en 1854 et 1872-I873, nous combinons les élèves «apprenant l'arithmétique simple» et «l'arithmétique composée». En I882, il n’y a plus qu'une seule rubrique intitulée «arithmétique».

4. RSIP 1854, Extrait des tableaux statistiques fournis par les inspecteurs d'école [...], I8-19.

5. RSIP 1863, Grand tableau statistique préparé d’après les rapports des inspecteurs, des commissaires et syndics d’école, etc., 60. Les données incluent les élèves du collège de Trois-Rivières.

6. RSIP 1872-1873, Récapitulation des grands tableaux statistiques préparés d'après les rapports des inspecteurs, des commissaires d'école, etc., 195-196. Les données incluent les élèves du collège de Trois-Rivières.

7. RSIP I882-1883, Récapitulation. Grand tableau statistique préparé par MM. les inspecteurs d'école [...], 198-199. Cumul des données pour les districts de David Lefebvre comprenant 8 localités du comté de Portneuf et de Ambroise Tétreault. Les données incluent les élèves du collège de Trois-Rivières.

8. RSIP I892-1893, Statistique générale concernant les élèves, d’après les bulletins des inspecteurs d’écoles [...], I84-|85. Cumul des données des comtés de Champlain, de Maskinongé et de Saint-Maurice, incluant la ville de Trois-Rivières. Le total des élèves provient de la Statistique générale des écoles, 180-183. Les données excluent les élèves du collège de Trois-Rivières.

9. RSIP 1898-1899, Statistique générale concernant les élèves [...], 209 et ss. Cumul des données sur les trois comtés et la ville de Trois-Rivières. Les données excluent les élèves du collège de Trois-Rivières. 
font partie soit d'une classe surpeuplée ou d'un groupe dont les parents n'achètent pas le matériel nécessaire. Lorsque la corporation fournit ellemême les ardoises, tous les élèves apprennent à écrire. Cette amélioration illustre non seulement un progrès dans l'enseignement de cette matière, mais indique un intérêt croissant pour l'école chez les parents et les commissaires, qui acceptent de fournir le matériel pédagogique requis. À la fin du siècle, tous les écoliers font l'apprentissage de l'écriture.

L'arithmétique n'est longtemps enseignée qu'à un tiers des élèves. Dès son entrée en fonction (1877-1878), Ambroise Tétreault s'étonne de voir si peu d'élèves apprendre à compter. «Le calcul, cette autre branche d'instruction si indispensable, est bien négligé, et est mal enseigné même dans un bon nombre d'écoles que je compte comme bonnes et passables ${ }^{54}$.»

Toutefois, quelques années plus tard, l'inspecteur Lefebvre estime que tous les élèves font du calcul mental, bien que beaucoup n'aient pas encore maîtrisé l'écriture. En outre, plus du tiers des écoliers de son district font de l'arithmétique écrite, c'est-à-dire qu'ils « savent poser un nombre quelconque» ou qu'ils apprennent une des quatre opérations de base ${ }^{55}$. Mais cette matière est moins enseignée, probablement à cause d'une formation déficiente des titulaires, comme l'observe A. Tétreault, signalant «que presque toutes les jeunes institutrices sont d'une faiblesse impardonnable [en calcul $]^{56}$ ". Peu à peu, cependant, les résultats s'améliorent puisqu’à la fin de la décennie 1880, un plus grand nombre d'enfants maitrisent le calcul «d'une manière pratique» ou suffisamment "pour leur permettre de faire leurs petites affaires» et ce, dans le plus grand nombre des écoles ${ }^{57}$. En 1892-1893, l'arithmétique est étudiée par $77 \%$ des élèves. À la fin du siècle, elle est enseignée à la totalité d'entre eux.

La géographie, pour sa part, reste longtemps négligée. À quelques reprises, Petrus Hubert souligne l'absence de cartes géographiques ou leur vétusté $^{58}$. De son côté, en 1877-1878, David Lefebvre se réjouit du fait que les corporations peuvent acheter une nouvelle série de cartes par l'entremise du dépôt de livres ${ }^{59}$. En 1882-1883, la proportion des élèves qui s'initient à

54. RSIP 1877-1878, Rapport de Ambroise Tétreault, 94.

55. RSIP 1882-1883, Rapport de David Lefebvre, 58 et ss.

56. RSIP 1882-1883, Rapport de Ambroise Tétreault, 147-148.

57. RSIP 1889-1890, Rapport de Ambroise Tétreault, 93.

58. RSIP, 1856, Rapport de Petrus Hubert, 168; et RSIP, 1871-1872, 37.

59. Le dépôt de livres, instauré par le département de l'Instruction publique en 1876, incitera les corporations scolaires à renouveler leurs fournitures scolaires. Ce service sera aboli trois ans plus tard. 
cette matière croît à $22 \%$. Un meilleur approvisionnement en cartes porte ses fruits ${ }^{60}$. À la fin du siècle, $69 \%$ des écoliers étudient la géographie.

La tenue des livres ne rallie que $3 \%$ des effectifs en 1863 . À la faveur des programmes de 1873 et de 1878, les élèves de la fin de l'élémentaire sont introduits à la tenue des livres en partie simple, ce qui porte à $12 \%$ le taux des écoliers étudiant cette matière en 1882-1883. Ils apprennent les rudiments de la comptabilité : faire des entrées précises, équilibrer des comptes, préparer des factures et des reçus ${ }^{61}$. Dans le programme de 1888 , il n'est plus question de cette matière à l'élémentaire; elle est intégrée au cours modèle, dans le programme des mathématiques. En 1898-1899, 7 \% des élèves étudient la tenue des livres, taux qui correspond assez bien à la population des élèves inscrits dans les écoles modèles et académiques.

En 1900, que possèdent les écoliers comme instruction au terme des deux premières années du cours élémentaire? Ils maîtrisent la lecture courante, avec la signification des mots tirés du livre d'apprentissage, ainsi que l'écriture sur papier; ils ont quelques rudiments de grammaire (voyelles, consonnes, accents, noms et adjectifs). Les cours de mathématiques couvrent la numérotation jusqu'à 10000 , les quatre règles simples, le calcul mental, les monnaies du pays et les tables de multiplication et de division. La géographie les a familiarisés avec leur environnement immédiat, y compris la circonscription, et quelques notions, apprises en troisième année, qui concernent la province de Québec et le reste du Canada. En troisième année de l'élémentaire, l'élève lit avec l'intonation convenable, connaît les verbes réguliers, fait de l'analyse grammaticale; il possède un cahier de devoirs journaliers, étudie les fractions et peut résoudre des problèmes pratiques, comme faire des comptes et des factures. Les connaissances usuelles comprennent des leçons sur l'agriculture. La quatrième et dernière année du cours élémentaire récapitule les enseignements de l'année précédente auxquels s'ajoutent de nouvelles matières : comptabilité domestique, géographie étendue aux continents et premiers essais de cartographie.

Ces constatations nous permettent de revenir sur les jugements émis par les inspecteurs concernant la qualité du personnel enseignant. Jusqu'en 1900, il ne s'est pas produit de changement significatif dans le degré d'instruction des institutrices en place. Et pourtant, l'enseignement des matières

60. RSIP 1886-1897. Dans son rapport, Ambroise Tétreault, précise que : «Les écoles sont toujours assez bien fournies de tout ce qu'il leur faut, sauf quelques rares exceptions. Pas une ne manque des cartes géographiques nécessaires. », 125.

61. Michel Allard et Bernard Lefebvre, op. cit., 151 et 162. 
a connu une amélioration notable. Comme le contenu des cours est, à toutes fins utiles, du ressort des enseignantes, il est permis d'avancer que ces dernières ont fourni des efforts appréciables malgré les carences de leur formation. Il est donc opportun, dans de futures recherches, de jeter un œil plus attentif sur les méthodes qu'elles ont utilisées. Les institutrices étaient le plus souvent issues du milieu où elles travaillaient et devaient adapter l'enseignement à ce milieu. L'étude de leurs approches et des valeurs qu'elles véhiculaient devient alors cruciale. Dans cette perspective, l'enseignante, loin d'être une entité passive du DIP ou de la commission scolaire, devient l'agent premier de la diffusion des connaissances. Telle est, en tout cas, l'hypothèse que nous aimerions formuler à partir du cas mauricien.

\section{CONCLUSION}

La scolarisation élémentaire en Mauricie entre 1850 et 1900 est d'abord l'histoire d'une institution qui s'implante partout sur le territoire. C'est aussi l'histoire d'une évolution lente puisqu'il aura fallu cinquante ans pour que l'école devienne non seulement le passage incontournable de la presque totalité des enfants âgés entre 5 et 13 ans, mais pour qu'elle devienne efficace dans l'enseignement des matières de base.

Cependant, ces résultats globaux sont affectés par l'absentéisme et par le retrait précoce des enfants. La plupart des jeunes des campagnes n'achèvent, encore en 1900, que deux des quatre années du cours élémentaire. En cela, les trois circonscriptions rurales se démarquent de Trois-Rivières et du reste du Québec. Parmi elles, c’est Maskinongé qui obtient les meilleurs résultats, suivie de Champlain. Dans Saint-Maurice, la plus démunie des trois sur le plan économique, la majorité des élèves n'achèvent que la première année du cours élémentaire. En outre, les études dans les écoles publiques après l'âge de 13 ans intéressent surtout les filles. En 1900, elles forment $68 \%$ des effectifs du "cours supérieur» surpassant ainsi la proportion des écolières québécoises $(57 \%)$ de cette catégorie.

Il ne fait pas de doute que la socio-économie est pour quelque chose dans ces résultats, en particulier dans les régions nouvellement habitées. Il existe aussi des préjugés face à l'école dans ce monde à majorité rurale. La double tendance que nous avons observée d'un progrès à long terme accompagné de lacunes persistantes explique sans doute les propos contradictoires tenus par bien des contemporains. Dans les années 1870, en effet, pour un inspecteur qui avance qu' «il n'est pas de si petit noyau de population fixé dans 
les défilés de nos montagnes, qui ne veuille avoir son école ${ }^{62}$ », il se trouve d'autres personnes pour estimer qu'il «ne faut pas tant d'instruction pour cultiver la terre ${ }^{63}$ ", tandis que plusieurs donnent la priorité aux corvées domestiques, au détriment d'une présence régulière en classe.

Il n'en reste pas moins que les progrès de la scolarisation deviennent plus évidents à compter des années 1880 . Cela revient pour une grande part au personnel enseignant, constitué en grande majorité par des institutrices. Elles n’ont, en général, aucune véritable formation en pédagogie. La fréquentation assidue de l'école de rang ou du village et la réussite des épreuves du bureau des examinateurs leur permettent d'acquérir un brevet d'enseignement. Tout de même, au fil des années, les institutrices parviennent à transmettre plus efficacement les matières de base. Leur travail a peut-être aussi convaincu les contribuables de la nécessité de s'intéresser davantage à l'école.

Le parcours de l'instruction publique en Mauricie est-il unique ou ressemble-t-il à celui d'autres régions du Québec? Nous avons, bien sûr, fait des comparaisons avec l'ensemble de la province, mais elles englobent le monde urbain où les conditions sont très différentes. L'état actuel des recherches régionales en éducation interdit de comparer ces résultats avec ceux d'autres parties du Québec. Des recherches ultérieures permettraient de faire d'utiles comparaisons et de mieux cerner les conditions encourageant l'éducation à prendre racine.

62. RSIP 1877-1878, Second rapport de David Lefebvre, 60.

63. ANQ, Correspondance reçue de Sainte-Geneviève, document $\mathrm{n}^{\circ}$ 2510, 1878 : lettre du secrétaire-trésorier Robert Trudel, en date du 24 septembre. 